\title{
POPULATION STUDY ARTICLE Co-occurrence of infantile hypertrophic pyloric stenosis and congenital heart defects: a nationwide cohort study
}

\author{
Bjarke Feenstra ${ }^{1}{ }^{1}$, Sanne Gørtz ${ }^{1}$, Marie Lund ${ }^{1}$, Mattis F. Ranthe ${ }^{1}$, Frank Geller ${ }^{1}$ and Mads Melbye ${ }^{1,2}$
}

BACKGROUND: Recent studies suggest that infantile hypertrophic pyloric stenosis (IHPS) and congenital heart defects (CHDs) may share some genetic risk factors, but little is known about the co-occurrence of the two conditions in patients.

METHODS: Our study cohort included 2,212,756 persons born in Denmark 1977-2013. We identified patients with IHPS and CHD in the National Patient Register. Using log-linear Poisson regression, we estimated the (incidence) rate ratios (RRs) comparing the rate of IHPS among children with a CHD diagnosis (exposed) and the rate among those without such a diagnosis.

RESULTS: Twenty-seven thousand three hundred and fifty-seven children in the cohort were diagnosed with CHD out of whom 85 developed IHPS ( $R R=2.62,95 \%$ confidence interval $(\mathrm{Cl}) 2.09-3.22]$ ). The results were similar for those with and without other congenital malformations, for preterm and term deliveries, and for both sexes. There was, however, a significant effect of calendar period $(P=.003)$. In the period 1977-1996, the RR of IHPS given a CHD diagnosis was $1.96(95 \% \mathrm{Cl} 1.41-2.64)$; in the period 1997-2014, the RR was 3.75 (95\% Cl 2.74-4.99).

CONCLUSION: CHD was associated with an increased risk of IHPS. Further research is needed to delineate molecular-level mechanisms that may affect both conditions.

Pediatric Research (2019) 85:955-960; https://doi.org/10.1038/s41390-019-0369-9

\section{INTRODUCTION}

Infantile hypertrophic pyloric stenosis (IHPS) is caused by progressive hypertrophy of the smooth muscle layer of the pyloric sphincter leading to obstruction of the gastric outlet to the duodenum. Symptoms typically appear in otherwise healthy infants 3-12 weeks after birth ${ }^{1}$ and include non-bilious projectile vomiting, dehydration, and weight loss. ${ }^{2}$ IHPS can be relieved permanently through surgical incision of the pyloric sphincter muscle, a safe and effective procedure introduced by Ramstedt more than a century ago. ${ }^{2}$

The incidence of IHPS among Caucasians is $1-3$ per 1000 live births ${ }^{3}$ and somewhat lower in other ethnic groups. ${ }^{1}$ Changes in incidence observed in several countries over the past decades ${ }^{4}$ provide evidence for the influence of environmental factors. However, IHPS also aggregates strongly in families, and a genetic predisposition to the condition is well established. ${ }^{3}$ Affecting 4-5 times as many boys as girls, IHPS was defined by Carter as a paradigm for the multi-factorial sex-modified threshold model of inheritance. ${ }^{5}$

Most IHPS cases occur as isolated entities. One large epidemiological study based on birth defects registered before 1 year of age found that out of almost 2000 IHPS cases in California, $>90 \%$ had no additional birth defects, another $8 \%$ had $\geq 1$ additional minor or major defects, and $<1 \%$ were identified with a specific clinical syndrome. ${ }^{1}$ Birth defects that have been reported to occur together with non-syndromic IHPS include renal and urological abnormalities ${ }^{1,6,7}$ and congenital heart defects, ${ }^{8}$ but results have been conflicting and it is unclear whether these observations reflect shared etiological factors. ${ }^{1,7,9}$
A better etiological understanding of IHPS carries the additional benefit of shedding light on possible comorbidities. Recent genetic association studies have identified sequence variants associated with isolated IHPS in or near the genes APOA1, MBNL1, EML4, BARX1, and NKX2-5. ${ }^{10-14}$ NKX2-5 encodes the homeobox transcription factor NKX2-5, which is essential for normal heart formation and development. ${ }^{15}$ Since mutations within NKX2-5 are known to be associated with different forms of congenital heart defects (CHDs), ${ }^{16}$ we aimed to investigate whether being diagnosed with a CHD also conferred risk of IHPS. Using nationwide health register data, we investigated the cooccurrence of the two disorders in the Danish population.

\section{METHODS}

Study Cohort

The study cohort included all live born singletons in Denmark from 1977 through 2013 and was identified using the Danish Civil Registration System. ${ }^{17}$ Since April 2, 1968, the Civil Registration System has recorded information on sex, date and place of birth, identity of parents, and continuously updated information on vital status and emigration of all residents in Denmark. Furthermore, each resident is assigned a unique personal identification number, which permits linkage of individual-level information among other Danish population-based registers.

Identification of IHPS cases

Information on IHPS status was obtained by linkage with the Danish National Patient Register, which contains nationwide

\footnotetext{
${ }^{1}$ Department of Epidemiology Research, Statens Serum Institut, 5 Artillerivej, DK-2300 Copenhagen S, Denmark and ${ }^{2}$ Department of Medicine, Stanford University School of Medicine, Stanford, CA, USA

Correspondence: Bjarke Feenstra (fee@ssi.dk)
}

Received: 2 November 2018 Revised: 1 March 2019 Accepted: 4 March 2019

Published online: 12 March 2019 
956

registration of all somatic inpatient discharge diagnoses and surgeries performed since 1977 as well as outpatient visits since 1995. ${ }^{18}$ IHPS cases were defined as children who in their first year of life had pyloromyotomy performed: up to December 1995 according to codes from the Danish Classification of Surgical Procedures (codes 418.40, 418.41, and 441.00) and, from January 1996, according to codes from the NOMESCO Classification of Surgical Procedures (codes KJDH60 and KJDH61). Date of IHPS diagnosis was defined as the date of hospitalization for IHPS surgery or, if a distinct admission or outpatient visit including a diagnosis code for IHPS (International Statistical Classification of Diseases (ICD) Eighth Revision [ICD8], codes 537.01, 750.19, and 784.29, ICD, Tenth Revision (ICD10), codes K31.1, K31.3, K31.8B, and Q40.0) preceded date of hospitalization for IHPS surgery, the date of the record containing the diagnosis code was used to define the date of IHPS diagnosis.

Identification of CHD cases

Information on CHD status was also obtained by linkage with the Danish National Patient Register, defining CHD cases as individuals who at any age were registered with any of the following diagnosis codes: ICD8, codes 746.00-747.59, 759.00-759.09; ICD10, codes Q20.0-Q26.9, Q89.3. Date of CHD diagnosis was defined as the date of the first hospital admission or outpatient visit with the record containing a CHD diagnosis code. Persons with CHDs were classified into sub-phenotypes by grouping specific ICD codes in a hierarchical fashion as described in earlier studies. ${ }^{19}$ Children born preterm with isolated patent ductus arteriosus (PDA) were not considered to be exposed in the analyses.

\section{Covariates}

Information on sex, date of birth, and birth order were obtained from the Danish Civil Registration System. Data on gestational age, birthweight, cesarean section, smoking, and maternal age were retrieved from the Danish Medical Birth Register. ${ }^{20}$ Small for gestational age was defined as the lower $10 \%$ percentile of estimated fetal weight according to the World Health Organization Fetal Growth Charts. ${ }^{21}$ Estimated fetal weights for gestational week 40 and later were not included in the charts. For those weeks, we instead used the observed birthweight distribution within strata of gestational age in the cohort. Information on congenital malformations was obtained by linkage with the Danish National Patient Register. The following diagnosis codes were used to define congenital malformations: ICD8, codes 740.00-759.99 (excluding 746.00-747.59, 759.00-759.09 [CHD], and 750.19 [IHPS]); ICD10, codes Q00-Q99, D215, D821, P350, P351, P371 (excluding Q40.0 [IHPS]; and other minor malformations as defined by EUROCAT (http://www.eurocat-network.eu/ content/EUROCAT-Guide-1.4-Section-3.2.pdf) and Q20.0-Q26.9, Q89.3 [CHD]).

\section{Statistical analysis}

Using log-linear Poisson regression, we estimated the (incidence) rate ratios (RRs) comparing the rate of IHPS among children with a CHD diagnosis (exposed) and the rate among those without such a diagnosis. Infants were followed from birth until 1 year, death, emigration, IHPS diagnosis, or December 31, 2014, whichever occurred first.

All RRs were adjusted for the following potential confounders: attained age, sex of cohort member, and calendar period (5-year intervals). Fetal CHD is associated with increased risk of preterm birth, ${ }^{22}$ intrauterine growth restriction, ${ }^{23}$ and cesarian section. ${ }^{24}$ Gestational age, small for gestational age, and cesarean section have also been associated with IHPS ${ }^{25}$ and these factors were therefore considered intermediary variables in our analyses. The natural direct effect of CHD on IHPS not mediated by these variables was estimated by the weighting method of Lange et al. ${ }^{26}$ as described in Supplemental Material. These analyses were made under the assumption of no unmeasured confounding of the intermediary variables and IHPS. To assess the effects of deviations from this assumption on our results, we performed a sensitivity analysis, as detailed in Supplemental Material. We used interaction terms to evaluate effect modifications by sex, congenital malformations, attained age, and calendar period before and after the significant decline in IHPS incidence seen in Denmark in the early 1990s. The effect modification analysis for other congenital malformations addresses the question of whether an association between CHD and IHPS could be attributed to a generalized and nonspecific tendency for birth defects to cluster. To further investigate this question, we compared the risk of IHPS among children with CHD (with or without another congenital malformation) to the risk of IHPS among children without CHD but with another malformation. ${ }^{27}$ Additional sensitivity analyses included adjustment for birth order, maternal age, and smoking, but this had very little effect on RR estimates (results not shown).

As CHD is present from birth, we chose to let all individuals with a diagnosis of CHD during follow-up be exposed from birth. We also carried out time-dependent sub-analyses: (1) where the children were exposed from the diagnosis of CHD (i.e., children with a CHD diagnosis before their IHPS diagnosis were considered as exposed and children with a CHD diagnosis after their IHPS diagnosis were considered as not exposed), and (2) the reverse situation in which the outcome was diagnosis of CHD and children were exposed from diagnosis of IHPS (i.e., children with an IHPS diagnosis before their CHD diagnosis were considered as exposed and children with an IHPS diagnosis after their CHD diagnosis were considered as not exposed). In the latter analysis, infants were followed until death, emigration, CHD diagnosis, or December 31, 2014, whichever came first. In the timedependent sub-analyses, cohort members with identical date of IHPS diagnosis and CHD diagnoses were considered to have IHPS first ( 15 children) if surgery for IHPS was performed within 12 days of hospital admission, and CHD first (1 child) if IHPS surgery took place $>12$ days after admission. In case it was not possible to determine the precise date of IHPS surgery (before 1996) and the admission was too long to determine an approximate date, the cohort members were considered to have IHPS first (4 children).

\section{RESULTS}

The study cohort included 2,212,756 persons born in Denmark in 1977-2013. Out of these, 25,529 were diagnosed with a CHD before 31 December 2014. A total of 3415 had surgery for IHPS during follow-up, out of which 85 had a CHD diagnosis. Table 1 shows characteristics of the cohort members with and without IHPS. The distribution of specific cardiac malformations in cohort members with and without IHPS is shown in Table 2. RRs of IHPS according to CHD diagnosis (exposure) are shown in Table 3. The overall RR estimate adjusting for age, sex, and calendar period was 2.62 [95\% confidence interval (CI) 2.09, 3.22]; $P<.0001)$. The RR estimate for the natural direct effect of CHD on IHPS not mediated by cesarean section, gestational age, and small for gestational age was 2.24 [95\% Cl 1.76, 2.85]).

There were no indications of effect modification by sex or having other congenital malformations. There was, however, a significant effect of calendar period $(P=.003)$. In the period 1977-1996, the RR of IHPS given a CHD diagnosis was $1.96[95 \% \mathrm{Cl}$ $1.41,2.64]$; in the period 1997-2014, the RR was 3.75 [95\% Cl 2.74, 4.99]. The effect of having a CHD was also significantly modified by attained age $(P=.001)$, with an RR estimate of $5.64[95 \% \mathrm{Cl}$ $3.60,8.38$ ] for age at IHPS diagnosis of $0-2$ weeks, and RR estimates around 2 for age at IHPS diagnosis of $\geq 3$ weeks.

In analyses addressing the possible scenario that an association between CHD and IHPS could be driven by generalized and nonspecific tendency for birth defects to cluster, we found that RR estimates did not differ between a stratum where the individuals 
Table 1. Characteristics of children without and with IHPS

\begin{tabular}{|c|c|c|}
\hline & $\begin{array}{l}\text { IHPS: no } \\
(n=2,209,341), \\
N(\%)\end{array}$ & $\begin{array}{l}\text { IHPS: yes } \\
(n=3415), \\
N(\%)\end{array}$ \\
\hline \multicolumn{3}{|l|}{ Sex } \\
\hline Males & $1,132,968(51.28)$ & $2806(82.17)$ \\
\hline Females & $1,076,373(48.72)$ & $609(17.83)$ \\
\hline \multicolumn{3}{|l|}{ Firstborn ${ }^{a}$} \\
\hline Yes & $908,411(44.46)$ & $1803(54.87)$ \\
\hline No & $1,134,573(55.54)$ & $1483(45.13)$ \\
\hline \multicolumn{3}{|l|}{ Gestational age, weeks ${ }^{b}$} \\
\hline $23-36$ & $95,447(4.44)$ & $261(7.97)$ \\
\hline $37-43$ & $1,985,135(92.38)$ & $2885(88.06)$ \\
\hline Unknown & $68,355(3.18)$ & $130(3.97)$ \\
\hline \multicolumn{3}{|l|}{ Small for gestational age ${ }^{\mathrm{b}}$} \\
\hline Yes & $207,964(9.68)$ & $400(12.21)$ \\
\hline No & $1,865,586(86.81)$ & $2737(83.55)$ \\
\hline Unknown & $75,387(3.51)$ & $139(4.24)$ \\
\hline \multicolumn{3}{|l|}{ Cesarean section ${ }^{\mathrm{b}}$} \\
\hline Yes & $297,217(13.83)$ & 645 (19.69) \\
\hline No & $1,776,441(82.67)$ & $2529(77.20)$ \\
\hline Unknown & $75,279(3.50)$ & $102(3.11)$ \\
\hline \multicolumn{3}{|c|}{ Maternal smoking in pregnancy ${ }^{c}$} \\
\hline Yes & $287,083(20.05)$ & $431(30.25)$ \\
\hline No & $1,084,726(75.76)$ & $926(64.98)$ \\
\hline Unknown & $59,906(4.18)$ & $68(4.77)$ \\
\hline \multicolumn{3}{|l|}{ Congenital heart defect } \\
\hline Yes & $25,444(1.15)$ & $85(2.49)$ \\
\hline No & $2,183,897(98.85)$ & $3330(97.51)$ \\
\hline \multicolumn{3}{|c|}{ Other congenital malformations } \\
\hline Yes & $132,756(6.01)$ & $363(10.63)$ \\
\hline No & $2,076,585$ (93.99) & $3052(89.37)$ \\
\hline Year of birth, mean (SD) & 1995 (10.48) & $1991(9.90)$ \\
\hline Maternal age, mean (SD) & $28.84(5.06)$ & $27.67(5.11)$ \\
\hline \multicolumn{3}{|c|}{$\begin{array}{l}\text { IPHS infantile hypertrophic pyloric stenosis } \\
\text { a The counts cover the years } 1977-2010 \text {, as data on parity was not available } \\
\text { for cohort members born in } 2011-2013 \\
\text { bThe counts cover the years } 1978-2013 \text {, as data on gestational age, small } \\
\text { for gestational age, and cesarean section was not available for cohort } \\
\text { members born in } 1977 \\
\text { 'The counts cover the years } 1991-2013 \text {, as data on maternal smoking was } \\
\text { not available for births before } 1991\end{array}$} \\
\hline
\end{tabular}

also had other congenital malformations $(\mathrm{RR}=2.29[95 \% \mathrm{Cl} 1.51$, 3.32]) and a stratum without other malformations ( $R R=2.43[95 \%$ $\mathrm{Cl} 1.85,3.11]$ ) as shown in Table 3. Further, when restricting the analysis to individuals who had CHD and/or another congenital malformation, the estimated RR of IHPS given a CHD diagnosis was 1.71 [95\% Cl 1.34, 2.16].

In the main analyses, we defined all individuals with a diagnosis of $\mathrm{CHD}$ during follow-up as being exposed from birth. However, we also carried out time-dependent sub-analyses with results shown in Table 4. First, we defined exposure only from the time of CHD diagnosis, resulting in an RR of 1.89 ([95\% Cl 1.24, 2.73]; $P$ $=.004)$. Second, we performed analyses where the outcome was diagnosis of CHD and children were exposed from diagnosis of IHPS; here the RR was 2.89 ([95\% Cl 2.20, 3.71] $P<.0001)$. Further stratification by time since exposure (diagnosis of CHD or IHPS, respectively) showed that RRs were elevated immediately
Table 2. Types of CHDs in individuals without and with IHPS

\begin{tabular}{lcc}
\hline $\begin{array}{l}\text { Type of congenital } \\
\text { heart defect }\end{array}$ & $\begin{array}{c}\text { CHD but not IHPS } \\
(n=25,444), N(\%)\end{array}$ & $\begin{array}{c}\text { CHD and also IHPS } \\
(n=85), N(\%)\end{array}$ \\
\hline APVR & $260(1.02)$ & $0(0.00)$ \\
AVSD & $1135(4.46)$ & $6(7.06)$ \\
Complex & $51(0.20)$ & $0(0.00)$ \\
Conotruncual & $2191(8.61)$ & $3(3.53)$ \\
Conotruncual+AVSD & $51(0.20)$ & $0(0.00)$ \\
Heterotaxy & $414(1.63)$ & $0(0.00)$ \\
IAA & $447(1.76)$ & $3(3.53)$ \\
Isolated PDA, at term & $1154(4.54)$ & $5(5.88)$ \\
Isolated PDA, unknown & $161(0.63)$ & $0(0.00)$ \\
LVOTO & $1700(6.68)$ & $4(4.71)$ \\
Unspecified diagnoses & $4484(17.62)$ & $14(16.47)$ \\
Other heart defect & $2698(10.60)$ & $8(9.41)$ \\
RVOTO & $925(3.64)$ & $5(5.88)$ \\
Septal+LVOTO & $205(0.81)$ & $0(0.00)$ \\
Septal+RVOTO & $245(0.96)$ & $0(0.00)$ \\
Septal, ASD & $3791(14.90)$ & $14(16.47)$ \\
Septal, ASD+VSD & $925(3.64)$ & $3(3.53)$ \\
Septal, VSD & $4607(18.11)$ & $20(23.53)$ \\
\hline A &
\end{tabular}

$A P V R$ anomalous pulmonary venous return, ASD atrial septal defect, AVSD atrioventricular septal defect, $C H D$ congenital heart defect, IAA interrupted aortic arch, IPHS infantile hypertrophic pyloric stenosis, LVOTO left ventricular outflow tract obstruction, PDA patent ductus arteriosus, RVOTO right ventricular outflow tract obstruction, VSD ventricular septal defect

(0-1 weeks) after exposure. However, elevated risks were also seen long after exposure: the RR of IHPS was $4.84[95 \% \mathrm{Cl} 1.20$, 12.7] when $>10$ weeks had passed since CHD diagnosis, and in the analysis with CHD as outcome, the RRs remained high up to 5 years after IHPS diagnosis.

To assess the robustness of our results, we conducted a number of sensitivity analyses. First, we explored whether the difference in RRs between the calendar periods 1977-1996 and 1997-2014 could be explained by different lengths of follow-up. Since we defined all individuals with a CHD diagnosis during follow-up to be exposed from birth, individuals in the calendar period 1997-2014 had less opportunity to be defined as exposed compared to the early period. Thus exposed individuals in the later period presumably included a relatively higher proportion of severe CHD cases diagnosed early in life. However, when analyzing the data with the additional requirement that $\mathrm{CHD}$ diagnoses had to be present within the first year of life, we still found a significant effect of calendar period $(P=.005)$ : in the period 1977-1996 the RR was 2.18 [95\% Cl 1.42,3.18] and in the period 1997-2014 the RR was 4.46 [95\% Cl 3.20, 6.04]. Second, we performed a sensitivity analysis where CHD diagnoses based only on outpatient clinics were excluded. This raised the RR estimate of IHPS given CHD diagnosis slightly to 2.85 [95\% Cl 2.21, 3.60] compared with 2.62 in the main analysis (Table 3 ). Third, in our main analysis preterm children with PDA were not considered exposed. It is conceivable that some term PDA cases could have had misclassified gestational age information (i.e., that they were actually born preterm) and some PDA cases had missing gestational age information. We therefore performed a sensitivity analysis where no PDA cases were considered exposed. This resulted in an RR estimate of 2.58 [95\% Cl 2.05, 3.20] close to 2.62 in the main analysis (Table 3). Fourth, in the time-dependent analyses it was not possible to unambiguously resolve the order of the two diagnoses for 20 individuals. We analyzed the data under 
Co-occurrence of infantile hypertrophic pyloric stenosis and congenital...

B Feenstra et al.

Table 3. Rate ratios of IHPS according to CHD diagnosis (exposure) overall and by sex of cohort member, gestational age, presence of other malformations, calendar period, and attained age at IHPS diagnosis

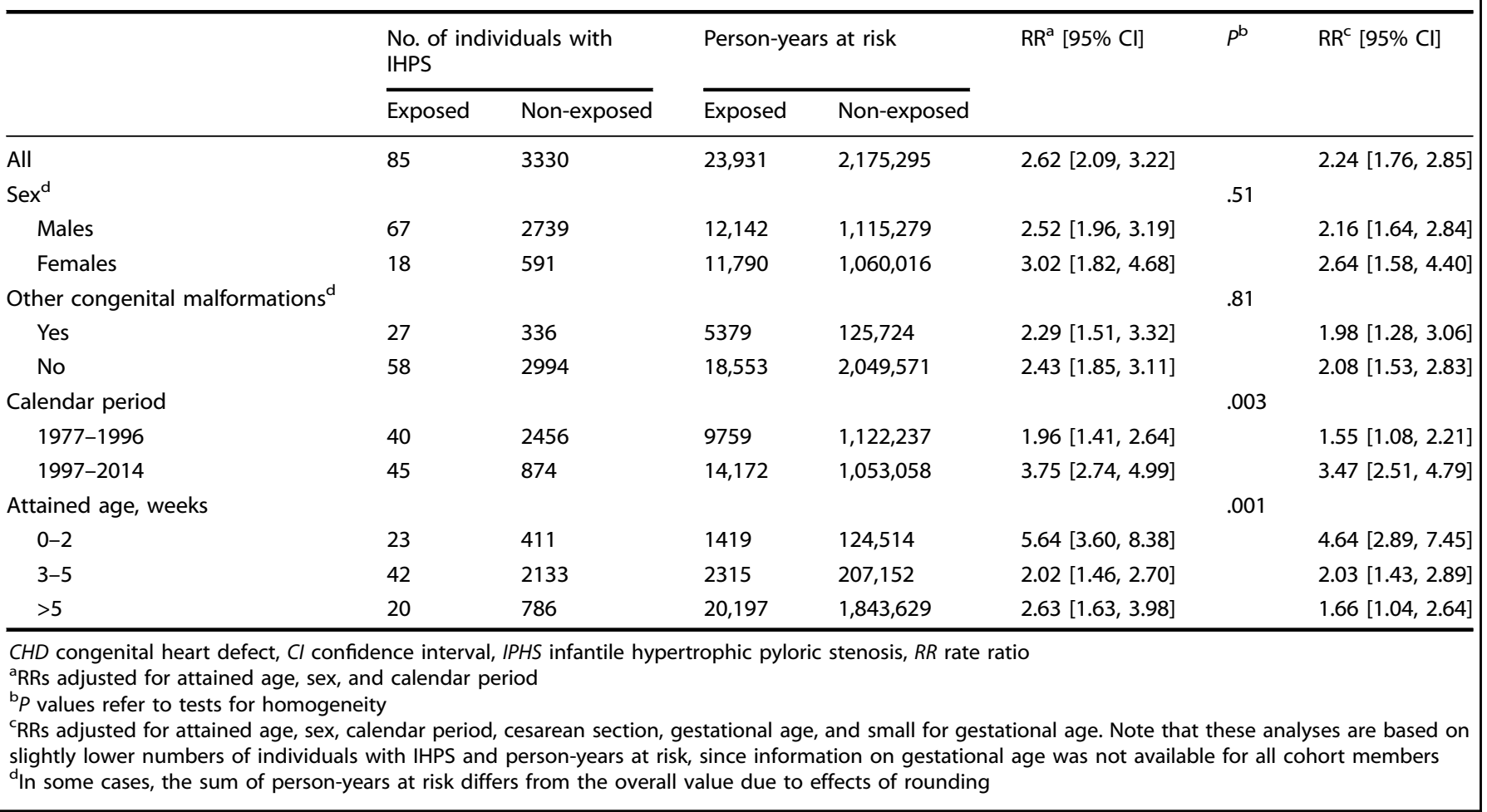

Table 4. Rate ratios of IHPS according to CHD diagnosis and of CHD according to IHPS diagnosis

\begin{tabular}{|c|c|c|c|c|}
\hline & No. of individuals with outcome & Person-years at risk & $\mathrm{RR}^{\mathrm{a}}[95 \% \mathrm{Cl}]$ & $P$ \\
\hline All exposed & 25 & 13,395 & $1.89[1.24,2.73]$ & 0.004 \\
\hline $0-1$ & 5 & 625 & $3.06[1.10,6.60]$ & \\
\hline $2-3$ & 5 & 605 & $1.22[0.44,2.62]$ & \\
\hline$>10$ & 3 & 10,363 & $4.84[1.20,12.7]$ & \\
\hline Non-exposed & 3390 & $2,185,832$ & 1 [referent] & \\
\hline \multicolumn{5}{|c|}{ Exposure: IHPS, outcome: CHD } \\
\hline All exposed & 57 & 68,694 & $2.89[2.20,3.71]$ & $<.0001$ \\
\hline \multicolumn{5}{|c|}{ Time since IHPS diagnosis } \\
\hline $2-5$ years & 9 & 9183 & $2.29[1.10,4.13]$ & \\
\hline$>5$ years & 6 & 53,086 & $0.90[0.36,1.83]$ & \\
\hline Non-exposed & 23,542 & $34,935,274$ & 1 [referent] & \\
\hline
\end{tabular}

two alternative classification schemes where (1) all ambiguous cases were classified as having had IHPS diagnosed first, or (2) all ambiguous cases were classified as having had CHD diagnosed first. Using scheme (1) changed the classification of one person, thus only affect the estimates slightly (IHPS given exposure of
CHD: RR 1.81 [95\% Cl 1.18, 2.64], CHD given exposure of IHPS: RR 2.94 [95\% Cl 2.25, 3.77]; compare with Table 4). Using scheme (2) changed the classification of 19 persons, resulting in greater change in the estimates (IHPS given exposure of CHD: RR 3.42 [95\% Cl 2.51, 4.53], CHD given exposure of IHPS: RR $1.98[95 \% \mathrm{Cl}$ 
1.42, 2.66]; compare with Table 4), but still resulting in high RR estimates in both directions. Finally, a sensitivity analysis for an unmeasured confounder of the mediator and IHPS relationship showed that such a confounder would have to have a very pronounced effect to substantially influence the results (see Supplemental Material for details). For example, a confounder with a prevalence of 0.1 and decreasing the risk of having at least one of the mediators 10 times as well as increasing the rate of IHPS 10 times resulted in a corrected estimate of the direct effect of 2.02 (see Supplemental Material).

\section{DISCUSSION}

In this nationwide cohort study, we investigated the cooccurrence of CHD and IHPS. Our analyses showed that patients with $\mathrm{CHD}$ were at 2.6-fold increased risk of IHPS. These findings are compatible with a small retrospective study that reported increased occurrence of CHD in 58 non-syndromic IHPS cases. ${ }^{8}$ Also, a data-driven text-mining study of electronic health records from $>300,000$ patients revealed an unsuspected association between IHPS and ventricular septal defect. ${ }^{28}$

The observed co-occurrence of IHPS and CHD suggests that some etiological factors may be shared between the two conditions. A tabulation of CHDs into specific types of malformations did not suggest a different distribution of CHDs in IHPS cases compared to the overall population known to have CHDs, although a larger number of exposed IHPS cases would be needed to fully address this question. One possible shared pathogenic mechanism between IHPS and CHD might involve regulation of the NK2 homeobox 5 (NKX2-5) gene, which plays an essential role in normal heart formation and development. ${ }^{15}$ In humans, a range of NKX2-5 mutations have been identified that cause CHDs. ${ }^{16}$ There is large heterogeneity in the clinical presentation of patients with NKX2-5 mutations; frequent malformations include atrial septal defect with or without atrioventricular block, ventricular septal defect, and tetralogy of Fallot. ${ }^{16}$ In addition to mutations in the coding region of NKX2-5, variants in upstream regulatory elements also show an association with CHD. ${ }^{29}$ In adults, NKX2-5 is almost exclusively expressed in cardiac tissue, ${ }^{30}$ but during embryonic development NKX2-5 is essential for the formation of pyloric sphincter muscle tissue. ${ }^{31}$ Several recent genetic studies have identified and confirmed a genomewide significant sequence variant for IHPS near NKX2-5, ${ }^{10-14}$ and follow-up experiments in a mouse model indicate that regulatory changes altering NKX2-5 expression could contribute to pathogenesis of IHPS. ${ }^{32}$ Another line of evidence suggesting possible shared pathways in IHPS and CHD etiology comes from studies of the forkhead-related transcription factor 1 (FOXF1) gene. Structural variation affecting FOXF1 is associated with $\mathrm{CHDs}^{33}$ and a recent study of familial IHPS identified a FOXF1 missense mutation segregating with disease trait in an extended pedigree with eight IHPS cases. $^{34}$ Further studies are, however, clearly required to illuminate possible common regulatory mechanisms involved in IHPS and CHD pathogenesis. Even if some etiological factors are confirmed to be shared between IHPS and CHD, it is important to emphasize that both conditions are very heterogeneous and are influenced by other environmental and genetic factors that are not shared.

Given the occurrence of IHPS as part of many clinical syndromes, some of which are also characterized by cardiac anomalies, $^{35}$ one might speculate whether our association findings could be driven by syndromic cases presenting with both IHPS and CHD. In a similar vein, it could be argued that our observed association might be attributable to a generalized and nonspecific tendency for birth defects to cluster. We addressed these scenarios by extracting information about other congenital malformations from the Danish National Patient Register thereby identifying all children with congenital malformations, including possible syndromic cases resulting from, e.g., chromosomal abnormalities. In stratified analyses, we found no difference in RR estimates between those who had other congenital malformations and those who did not (Table 3). Moreover, when comparing children with $\mathrm{CHD}$ to only those with at least one other congenital malformation, and thus having approximately the same "load" of malformations, the RR was still elevated. While these results do not rule out a possible impact of generalized clustering of malformations or syndromic cases on our RR estimates, such factors are unlikely to fully account for the increased co-occurrence of IHPS and CHD. The RR estimates were the highest in the time period 1997-2014 (Table 3), which is after a significant decline in IHPS incidence in Denmark. ${ }^{36}$ The decline is most likely explained by changes in environmental factors rather than in genetic changes within the population. Therefore, we speculate that the relative influence of genetic factors in IHPS cases was largest in the most recent time period and that the common genetic effects on IHPS and CHD risk therefore were more pronounced than in 1977-1996. For example, bottle-feeding, an established environmental IHPS risk factor, ${ }^{37}$ was more common in the early period compared to the late period, due to increasing percentages of mothers breast feeding their infants during the $1990 \mathrm{~s}^{38}$

Strengths of this study include the use of a large populationbased cohort of $>2$ million children and the use of nationwide health and population registers, avoiding or minimizing the risks of recall bias, selection bias, and loss to follow-up. We obtained information on age and sex from the Danish Civil Registration System, which is considered close to complete. ${ }^{17}$ Information on gestational age, birthweight, and cesarean sections was obtained from the Danish Medical Birth Register. A validation study showed good agreement between information from medical records and data in the Danish Medical Birth Register except for a slight overestimation of gestational ages in the register. ${ }^{39}$ Although CHD, IHPS, and congenital malformation diagnoses in the National Patient Register have not been validated systematically, one study found an overall positive predictive value of $89 \%$ for a first time diagnosis with a cardiac malformation. ${ }^{40}$ Furthermore, hospital discharge diagnoses are mandatory and recorded for the entire country, and surgery codes in particular are considered accurate and well-recorded. ${ }^{18}$

The study also had limitations. The mediation analysis was conducted under the assumption of no unmeasured confounders of the mediators cesarean section, gestational age, and small for gestational age and the outcome IHPS. We cannot rule out the existence of such a confounder, but in a sensitivity analysis, we found that a very strong confounder would be needed to substantially influence the results. Also, as described in the Methods section we chose to assign the exposure of CHD from time of birth to individuals with a CHD diagnosis, regardless of age at diagnosis. This was done since CHD is not always diagnosed at birth but is per definition present at birth. As such, the exposure in our main analysis was conditioned on a future registration, which may or may not appear in the register before the IHPS diagnosis. However, in additional analyses we addressed this potential limitation, by examining (1) the RR given exposure time starting at CHD diagnosis and (2) reversing exposure/outcome, producing RRs for risk of CHD given an exposure of IHPS. In the main analysis, where some exposure time was conditioned on a future event the overall RR was 2.62, in sub-analysis (1) the RR was 1.89 , and in setup (2) the RR was 2.89 , all with highly significant $P$ values (Table 4 ).

It could also be argued that being hospitalized for CHD may lead to more intense surveillance for other conditions and thereby increase the probability of being diagnosed with IHPS. It should be noted, however, that IHPS is a life-threatening condition if the child is not treated. Thus, although it is conceivable that more intense surveillance of CHD patients would lead to earlier diagnosis of IHPS, we find it unlikely that some IHPS cases escaped attention altogether. Conversely, having had surgery for 
IHPS could also increase the risk of getting a CHD diagnosis through more intense screening for CHD. However, we still observed an elevated RR of 1.89 when requiring the CHD diagnosis to have come first (Table 4). Also, as seen in the timedependent analysis with IHPS as exposure and CHD as outcome, the RRs remain elevated up to 5 years after IHPS diagnosis (Table 4). Together, these observations make it unlikely that ascertainment bias could account for all the increase in risk.

In conclusion, we found that CHD was associated with a 2.6-fold increased risk of IHPS, suggesting that these conditions may share common etiological factors. Further studies are needed to identify such factors and characterize their molecular-level role in disease pathogenesis.

\section{ACKNOWLEDGEMENTS}

This work was partially funded by the Danish Medical Research council (DFF 400400512) and was also supported by an Oak Foundation Fellowship (grant number OCAY12-319) to B.F. and a PhD study grant from the University of Copenhagen to M.L.

\section{AUTHOR CONTRIBUTIONS}

B.F. contributed to the conceptualization and design of the study, the acquisition of data, the interpretation of results, and the initial drafting of the manuscript; S.G. contributed to the design of the study, data analysis and interpretation of results, and reviewed and revised the manuscript; M.L., M.F.R., F.G., and M.M. contributed to the design of the study, the acquisition of data, the interpretation of results, and reviewed and revised the manuscript. All authors approved the final manuscript as submitted.

\section{ADDITIONAL INFORMATION}

The online version of this article (https://doi.org/10.1038/s41390-019-0369-9) contains supplementary material, which is available to authorized users.

Competing interests: The authors declare no competing interests.

Ethics: The study was approved by the Danish Data Protection Agency. In Denmark, ethics committee approval is not required for register based studies.

Publisher's note: Springer Nature remains neutral with regard to jurisdictional claims in published maps and institutional affiliations.

\section{REFERENCES}

1. Schechter, R., Torfs, C. P. \& Bateson, T. F. The epidemiology of infantile hypertrophic pyloric stenosis. Paediatr. Perinat. Epidemiol. 11, 407-427 (1997).

2. Spicer, R. D. Infantile hypertrophic pyloric stenosis: a review. Br. J. Surg. 69, 128-135 (1982)

3. Krogh, C. et al. Familial aggregation and heritability of pyloric stenosis. JAMA $\mathbf{3 0 3}$, 2393-2399 (2010)

4. Pedersen, R. N., Garne, E., Loane, M., Korsholm, L. \& Husby, S. Infantile hypertrophic pyloric stenosis: a comparative study of incidence and other epidemiological characteristics in seven European regions. J. Matern. Neonatal Med. 21, 599-604 (2008).

5. Carter, C. O. The inheritance of congenital pyloric stenosis. Br. Med. Bull. 17, 251-254 (1961).

6. Atwell, J. D. \& Levick, P. Congenital hypertrophic pyloric stenosis and associated anomalies in the genitourinary tract. J. Pediatr. 16, 1029-1035 (1981).

7. Applegate, M. S. \& Druschel, C. M. The epidemiology of infantile hypertrophic pyloric stenosis in New York State, 1983 to 1990. Arch. Pediatr. 149, 1123-1129 (1995).

8. Mehta, A. V. \& Ambalavanan, S. K. Infantile hypertrophic pyloric stenosis and congenital heart disease: an under-recognized association. Tenn. Med 90, 496-497 (1997).

9. Fernbach, S. K. \& Morello, F. P. Renal abnormalities in children with hypertrophic pyloric stenosis--fact or fallacy? Pediatr Radiol. 23, 286-288 (1993).

10. Feenstra, B. et al. Common variants near MBNL1 and NKX2-5 are associated with infantile hypertrophic pyloric stenosis. Nat. Genet. 44, 334-337 (2012).

11. Everett, K. V. \& Chung, E. M. Confirmation of two novel loci for infantile hypertrophic pyloric stenosis on chromosomes 3 and 5. J. Hum. Genet. 58, 236-237 (2013).

12. Feenstra, B. et al. Plasma lipids, genetic variants near $A P O A 1$, and the risk of infantile hypertrophic pyloric stenosis. JAMA 310, 714-721 (2013).
13. Feng, Z., Liang, P., Li, Q., Nie, Y. \& Zhang, Y. Association between NKX2-5 rs 29784 and infantile hypertrophic pyloric stenosis in Chinese Han population. Int. J. Clin. Exp. Med. 8, 2905-2910 (2015)

14. Fadista, J. et al. Genome-wide meta-analysis identifies BARX1 and EML4-MTA3 as new loci associated with infantile hypertrophic pyloric stenosis. Hum. Mol. Genet. 28, 232-240 (2019).

15. Fu, Y., Yan, W., Mohun, T. J. \& Evans, S. M. Vertebrate tinman homologues XNkx2-3 and $\mathrm{XNk \times 2-5}$ are required for heart formation in a functionally redundant manner. Development 125, 4439-4449 (1998).

16. Reamon-Buettner, S. M. \& Borlak, J. NKX2-5: an update on this hypermutable homeodomain protein and its role in human congenital heart disease (CHD). Hum. Mutat 31, 1185-1194 (2010).

17. Pedersen, C. B., Gotzsche, H., Moller, J. O. \& Mortensen, P. B. The Danish Civil Registration System. A cohort of eight million persons. Dan. Med. Bull. 53, 441-449 (2006)

18. Schmidt, M. et al. The Danish National patient registry: a review of content, data quality, and research potential. Clin. Epidemiol. 7, 449-490 (2015).

19. Botto, L. D., Lin, A. E., Riehle-Colarusso, T., Malik, S. \& Correa, A. Seeking causes: classifying and evaluating congenital heart defects in etiologic studies. Birth Defects Res. A Clin. Mol. Teratol. 79, 714-727 (2007).

20. Bliddal, M., Broe, A., Pottegård, A., Olsen, J. \& Langhoff-Roos, J. The Danish Medical Birth Register. Eur. J. Epidemiol. 33, 27-36 (2018).

21. Kiserud, T. et al. The World Health Organization fetal growth charts: a multinational longitudinal study of ultrasound biometric measurements and estimated fetal weight. PLoS Med. 14, e1002220 (2017).

22. Laas, E. et al. Preterm birth and congenital heart defects: a population-based study. Pediatrics 130, e829-e837 (2012).

23. Malik, S., Cleves, M. A., Zhao, W., Correa, A. \& Hobbs, C. A. Association between congenital heart defects and small for gestational age. Pediatrics 119, e976-e982 (2007).

24. Liu, C. et al. Obstetric and perinatal outcomes in pregnancies with isolated foetal congenital heart abnormalities. J. Matern. Neonatal Med. 3, 1-8 (2018).

25. Krogh, C. et al. Pre- and perinatal risk factors for pyloric stenosis and their influence on the male predominance. Am. J. Epidemiol. 176, 24-31 (2012).

26. Lange, T., Vansteelandt, S. \& Bekaert, M. A simple unified approach for estimating natural direct and indirect effects. Am. J. Epidemiol. 176, 190-195 (2012).

27. Prieto, L. \& Martínez-Frías, M. L. Epidemiological analysis of the association between two congenital anomalies in the same child: a method for adjusting nonspecific clustering. Am. J. Med. Genet. 62, 61-67 (1996).

28. Hanauer, D. A., Rhodes, D. R. \& Chinnaiyan, A. M. Exploring clinical associations using "-omics" based enrichment analyses. PLoS ONE 4, e5203 (2009).

29. Pang, S. et al. Genetic and functional analysis of the NKX2-5 gene promoter in patients with ventricular septal defects. Pediatr. Cardiol. 33, 1355-1361 (2012).

30. Kasahara, H., Bartunkova, S., Schinke, M., Tanaka, M. \& Izumo, S. Cardiac and extracardiac expression of Csx/Nkx2.5 homeodomain protein. Circ. Res. 82, 936-946 (1998)

31. Smith, D. M. \& Tabin, C. J. BMP signalling specifies the pyloric sphincter. Nature 402, 748-749 (1999).

32. Udager, A. M. et al. Proper development of the outer longitudinal smooth muscle of the mouse pylorus requires Nkx2-5 and Gata3. Gastroenterology 146, 157-165 (2014).

33. Stankiewicz, P. et al. Genomic and genic deletions of the FOX gene cluster on $16 q 24.1$ and inactivating mutations of FOXF1 cause alveolar capillary dysplasia and other malformations. Am. J. Hum. Genet. 84, 780-791 (2009).

34. Everett, K. V., Ataliotis, P., Chioza, B. A., Shaw-Smith, C. \& Chung, E. M. K. A novel missense mutation in the transcription factor FOXF1 cosegregating with infantile hypertrophic pyloric stenosis in the extended pedigree linked to IHPS5 on chromosome 16q24. Pediatr. Res. 81, 632 (2017).

35. Peeters, B., Benninga, M. A. \& Hennekam, R. C. Infantile hypertrophic pyloric stenosis--genetics and syndromes. Nat. Rev. Gastroenterol. Hepatol 9, 646-660 (2012).

36. Nielsen, J. P., Haahr, P. \& Haahr, J. Infantile hypertrophic pyloric stenosis. Decreasing incidence. Dan. Med. Bull. 47, 223-225 (2000).

37. McAteer, J. P., Ledbetter, D. J. \& Goldin, A. B. Role of bottle feeding in the etiology of hypertrophic pyloric stenosis. JAMA Pediatr. 167, 1143-1149 (2013).

38. Yngve, A. \& Sjostrom, M. Breastfeeding in countries of the European Union and EFTA: current and proposed recommendations, rationale, prevalence, duration and trends. Public Health Nutr. 4, 631-645 (2001).

39. Kristensen, J., Langhoff-Roos, J., Skovgaard, L. T. \& Kristensen, F. B. Validation of the Danish Birth Registration. J. Clin. Epidemiol. 49, 893-897 (1996).

40. Jepsen, B., Jepsen, P., Johnsen, S. P., Espersen, G. T. \& Sørensen, H. T. Validity of diagnoses of cardiac malformations in a Danish population-based hospital-discharge registry. Int J. Risk Saf. Med. 18, 77-81 (2006). 